\title{
Mapping Application Requirements to Cloud Resources
}

\author{
Yih Leong Sun, Terence Harmer, Alan Stewart, and Peter Wright \\ The Queen's University of Belfast, \\ University Road, Belfast, BT7 1NN, Northern Ireland, UK \\ \{ysun05, t.harmer, a.stewart,p.wright\}@qub.ac.uk \\ http://www.qub.ac.uk
}

\begin{abstract}
Cloud Computing has created a paradigm shift in software development. Many developers now use the Cloud as an affordable platform on which to deploy business solutions. One outstanding challenge is the integration of different Cloud services (or resources), offered by different Cloud providers, when building a Cloud-oriented business solution. Typically each provider has a different means of describing Cloud resources and uses a different application programming interface to acquire Cloud resources. Developers need to make complex decisions involving multiple Cloud products, different Cloud implementations, different deployment options, and different programming approaches. In this paper, we propose a model for discovering Cloud resources in a multi-provider environment. We study a financial use case scenario and suggest the use of a provider-agnostic approach which hides the complex implementation details for mapping the application requirements to Cloud resources.
\end{abstract}

Keywords: Cloud Computing, Cloud Programming Model, Resource Discovery.

\section{Introduction}

The emergence of Cloud Computing is creating a paradigm shift in software development. The International Data Corporation (IDC) published a research report in 2010 forecasting that the estimated spending on Public Cloud services will grow from $\$ 16.5$ billion in 2009 to over $\$ 55$ billion in 2014 [24]. The first wave of Public Cloud services was dominated by Software-as-a-Service (SaaS) applications, which contributed to $49 \%$ of Public Cloud spending in 2009. However, forecasts suggest that an increasing share of services will be provided by Platform-as-a-Service (PaaS), 16\%, and Infrastructure-as-a-Service (IaaS), 20\%, in 2014. A growing number of developers are using Cloud as the most affordable platform on which to deploy business solutions [25].

Cloud Computing offers many benefits to businesses. The consumption of Cloud services is usually on a pay-as-you-use pricing model. This can significantly reduce the upfront cost of purchasing software licenses or infrastructure hardware. It provides great flexibility for businesses to scale resources according to demand. In addition to that, the advantages of Cloud Computing includes 
dynamic resource provisioning, efficient multi-tenancy and server utilization which brings environmental benefits by reducing energy consumption and carbon emission 18.

There has been a significant growth in the number of Cloud service providers offering on-demand software services, application, storage and compute resources in the market. Providers may have different application programming interfaces to obtain and configure required Cloud services. From the application developer's point of view, there is a challenge to assemble Cloud services together, from various Cloud providers, in order to build a complete Cloud-oriented business solution. Application developers are not only interested in compute resources, such as CPU, memory, operating system type but are also interested in integrating other Cloud services, such as storage, database, messaging and communication services. When building a Cloud-oriented application, a developer must take into account requirements such as fault-tolerance, self-healing, self-management as well as the cost of Cloud services.

There is a need to develop an advanced high-level programming model for building Cloud-oriented business solutions in a multi-provider environment. The high-level model should enable cross-Cloud implementations and avoid a lowlevel technical restriction to a single Cloud provider. In this paper, we describe the concept of using a constraints-based model to map an application to a set of Cloud resources, which are to be used to execute the application, in a multiprovider environment. It should provide an abstract software layer that excludes implementation details from the issue of selecting Cloud resources.

\subsection{Paper Organisation}

The remainder of this paper is organised as follows. In section 2, we present a brief summary of our study on the management of Cloud resources. In section 3 , a financial sector use case scenarios is presented. In section 4 , we describe our proposed resource discovery model. In section 5, we present a prototype demonstrator. In section 6 , we study some related work. Section 7 suggests future work.

\section{Cloud Resource Management}

There are numerous Cloud management toolkits which manage and control infrastructure resources across multiple Cloud provider or virtualisation environments. These toolkits provide functionality for managing compute resources on the Cloud. Eucalyptus [6] provides an EC2-compatible programming interface and one-stop management console to manage hybrid clouds and private clouds with mixed hypervisor environments (XEN, KVM, vSphere, ESX, ESXi). It supports features such as storage integration, monitoring and auditing. OpenNebula [15] provides a standard-based open source cloud computing toolkit for building private, public or hybrid clouds using an interface which enables dynamic deployment and reallocation of virtual machines. Haizea [7] is an open source resource leasing manager which can be used as a VM scheduler for OpenNebula to 
provide leasing capabilities, such as resource reservation. Nimbus [10] is an open source toolkit that turns a cluster into an IaaS cloud. The Nimbus cloud client allows provisioning of compute nodes with a leasing model based on Amazon EC2 interface.

Most of the resource management toolkits in the market offer useful management features, however, they do not provide a search capability for Cloud resources that satisfy application's requirements. A developer needs to have preexisting knowledge of the infrastructure capabilities and pricing structures offered by different providers before using these toolkits to allocate infrastructure resources. Moreover, most of these toolkits are focused at the infrastructure layer, i.e. compute resources or virtual machines. There are a small number of toolkits for managing applications, such as, Message Queue Service [1164], Storage Service [123] and Database Service [2 5:11].

\section{Financial Use Case Scenarios}

In [30], an SLA (Service Level Agreement) focused financial services infrastrucutre framework is described. Two financial business scenarios are described. One of the financial business use cases requires the application developer to build a financial application to revalue a customer's stock or portfolio on a day to day basis in order to comply with internal market risk controls and national regulatory requirements. The execution of such application is very complex and requires computational intensive techniques. It requires high-end compute resources and large storage space. Extra compute power is needed, usually at short notice, if several large portfolios are to be valued at the same time. It needs an application platform to host a specific software bundle. It needs access to market data from a market feed services, such as Bloomberg market data feed. And one of the regulatory requirements is that data can not be held or transmitted outside the UK.

The other business use case presented in the paper is the building of a financial application that integrates different remote services and has the potential to run on multiple remote compute resources. It requires access to third-party financial services and compute resources on demand in order to run a financial market back-test simulation. It needs to pull historical data from different stock exchanges, such as London and New York. To ensure data quality and integrity, historical data needs to be verified and audited by a third-party data verification service. The verified data is fed into the financial application which runs on multiple remote compute resources. Large storage space is required to keep the historical data, verified data and the simulation result for further analysis. It must guarantee a low response time within a certain time range in the day. In addition, it has a maximum budget per month for running such application.

\subsection{Application Requirement's Challenges}

The financial sector is a challenging domain which requires high data volumes and rapid data processing with stringent security and confidentially requirements. 
Financial applications depends heavily on process and data intensive computations. It is highly regulated with national and international regulatory requirements. From the business use case scenarios described above, we identify the following key challenges when implementing a financial application in the Cloud:

- Performance Requirement. In the financial services sector, high availability of resources is at primary important. To ensure infrastructure resilience, multiple identical mirror infrastructures must be provisioned. It needs a very fast automatic mechanism to discover and re-provision resources when failure is detected.

- Compliance Requirement. Legislation regulation requirements are essential to the finance sector. A financial application must ensure the data quality, integrity and confidentiality in order to comply with the national and international regulations.

\section{Using Constraints to Discover Cloud Resources}

It is highly desirable to develop a novel programming model adhering to the service-oriented nature of Cloud Computing, allowing Cloud-oriented application to be constructed rapidly. It is proposed that a Cloud programming model provides a means of reasoning about user requirements or constraints (Fig. 1). In this paper, we propose a method for mapping an application with an associated set of constraints to a set of resources, that could be used to support the application. Examples of constraints include (but not limited to) the followings:

- Hardware constraints. Does the application have any hardware requirements; if so, how can we select a Cloud resource that meet the requirements.

- Software constraints. Does the application utilise any software; if so, is the software offered in a Cloud or must it be pre-installed by the user on an application platform.

- Storage constraints. Does the application need a storage space to keep data such as files or raw logs; if so, are these files stored in the Cloud.

- Data constraints. Does the application utilise a database; if so, is the database in a Cloud.

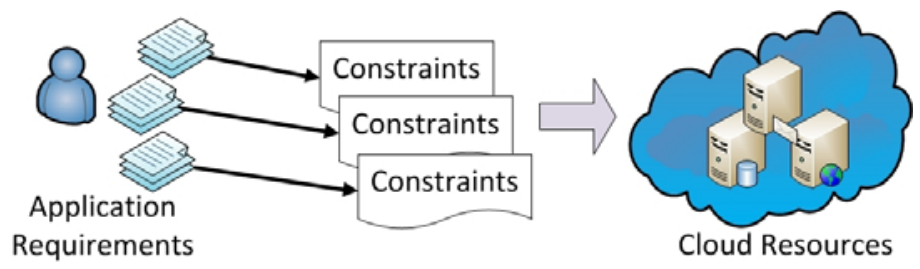

Fig. 1. Mapping Application Requirements to Cloud Resources 
- Security constraints. Does the application have any security or trust concerns; if so, is this security feature available from any Cloud provider.

- Performance constraints. Does the application need to fulfil a Quality of Service (QoS) or Service Level Agreement (SLA) requirement; if so, can a set of Cloud resources be found to meet the QoS or SLA.

- Cost constraints. Does the application have a budget or cost restriction; if so, can a set of Cloud resources be acquired within this budget.

- Compliance constraints. Does the application need to abide by any regulator or compliance requirement; if so, can a set of Cloud resources be found to meet the legal requirement.

Constraints can be simple, such as bandwidth of 1 gigabit, storage resilience of at least $99.9999 \%$, geographical location (resources must be in the UK), or business regulatory (must be in a TIA942 compliant datacentre).

As a simple example application consider a server which will host a large database. The resource must have at least $4 \mathrm{CPU}$ cores, $8 \mathrm{~GB}$ of RAM and $1 \mathrm{~TB}$ of local disk space. A more interesting example would be the provision of a financial infrastructure. Two identical mirror infrastructures must be provisioned, ideally widely separated, in order to ensure high resilience and high availability. The infrastructure being provisioned must:

- enforce financial regulatory requirements that the data must not be held or transmitted outside the UK;

- enforce performance requirements for a demanding application which requires high availability $(99.9999 \%)$ of compute power and data storage;

- balance the separation of the mirrored infrastructures against the performance need to keep the response time as low as possible (low latency).

The selection of resources must fulfil the regulatory requirements and balance the functional requirements required for the resources and the non-functional requirements required for high resilience.

Using a constraints-based model, the above example could be expressed as follow:

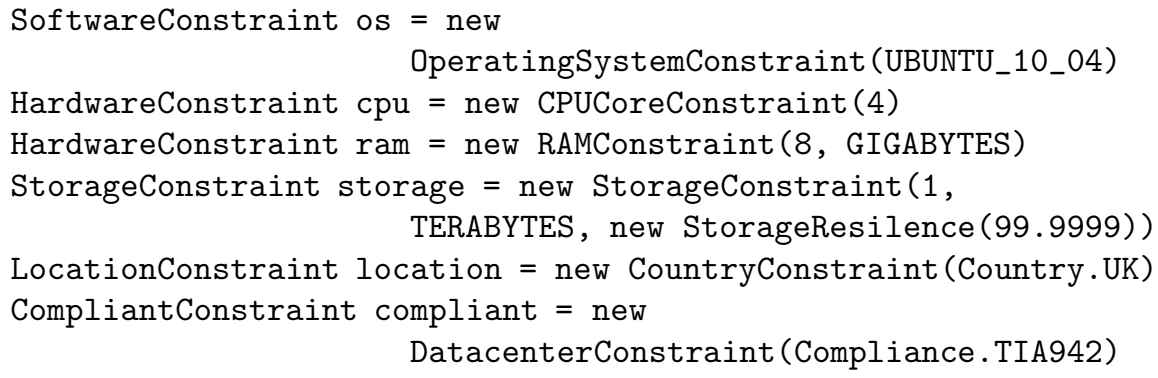

Resource [] resources $=$ FindResourcesByConstraints (os, cpu, ram, storage, location, compliant) 
It is necessary to have a clear description of Cloud resources in order to map the constraints to resources. Cloud resources can be described using semantic web techniques 1929 . Once the resources are defined, it is possible to translate the application requirements into constraints, which can be mapped to resource descriptions.

\section{Demonstrator}

We have implemented a simple prototype using the proposed constraints-based resource discovery model. In this prototype, Cloud resources are restricted to infrastructure compute resources (virtual machines) and storage resources (remote file storage) only. Amazon AWS and internal hardware infrastructures are used in this prototype to provision the resources. Following the approach of previous work [23, Cloud resources are described in terms of Resource Template with different attributes such as hardware specification, operating system type, storage capacity and datacenter location. Application requirements are translated into a set of constraints. In our experiment, we apply different hardware constraints (cpu, memory), software constraints (operating system), storage constraints (storage space) and regulatory constraints (geographical location) to search for viable resources. The performance of the demonstrator depends on the set of constraints being supplied. For example, searching resources in the EU region is much faster than searching for resources across all geographical regions. The experiment results show that the demonstrator can search and provision a set of Cloud resources that satisfy the application's requirements, using the proposed constraints-based model, in a multi-provider environment.

\section{Related Works}

The RESERVOIR project [28] presents an Open Federated Cloud Computing architecture that allows service providers to be grouped together so as to create a seemingly infinite pool of compute resources. The model separates the functional roles of service providers from infrastructures. Service providers need not necessarily own computational resources but may lease resources from infrastructure providers. This provides a clean separation of responsibilities and hides low level infrastructure details from the service providers. The RESERVOIR proposes a service definition manifest to specify the application configurations-using a service specification language 22 based on the DMTF's Open Virtualisation Format (OVF) standard [14. The service manifest includes VM details, application settings and deployment settings. In contrast to the RESERVOIR approach, we prefer a high-level constraints model which provides an application-focused (user-centric) rather than a provider-focused view of resources.

Aneka [31] is a PaaS (Platform as a Service) type of middleware for deploying Cloud applications. It provides a platform and framework for developing distributed applications on the Cloud. Aneka is being developed on top of EMCA 
Common Language Infrastructure [17. It requires the application to be implemented on .NET Framework 8] or Mono 9] only. A Platform Abstraction Layer is introduced in Aneka to encapsulate all of the platform dependencies on the hosting platform behind a common interface. An Aneka Container, the basic deployment unit of Aneka Cloud, supports different programming models including Task, Distributed Thread and MapReduce 20.

Mosaic [21 26 27. project intends to develop an open-source Cloud application programming interface and a platform for developing applications in a multi-Cloud environment. It studies patterns from related architectures (serviceoriented, parallel computing) and proposes a few Cloud Computing patterns such as Data storage and Multi-tenancy pattern. It proposes a resource negotiation solution based on software agents and semantic data processing. It presents a few functionalities that should be exposed by the Mosaic's API at the Storage, Communication, Monitoring and Provisioning level. It aims to provide a generic Cloud API by extending the OGF OCCI [13] proposal. It proposes an application model in terms of Cloud Building Block which can be a Cloud Resource under the Cloud provider control or a Cloud Component controlled by the application user. Mosaic is still under development. The first version of API will be made available in autumn 2011 and the full platform in another two years.

\section{Conclusion and Future Works}

Cloud application developers currently need to deal with a complex choice among multiple Cloud products, different Cloud implementations, different deployment options, and different programming approaches. Our proposed model is intentionally simple and attempts to reflect an ideal for the application user who wishes to specify services or resources without knowing the internal behaviour of the underlying provider - insulating the user from the different provider approaches and frequent changes in the underlying APIs. This approach means that the application can take a dynamic, commodity approach to service usage. A Cloud-oriented application can be deployed and scaled according to application and system constraints, stay within a strict budget and be portable between all available Cloud providers.

Most of the concepts described in this paper and the prototype demonstrator are still in preliminary stage. This research will serve as a base for future studies of a standard programming model for developing applications in the Cloud. We aim to develop a provider-agnostic mechanism in searching for Cloud resources in a multi-provider environment. The ability to map an application's requirements to a set of Cloud resources, based on a constraints-based model, can narrow the viable choices and allow automatic discovery of resources during application runtime.

\section{References}

1. Amazon Simple Queue Service (Amazon SQS), http://aws.amazon.com/sqs

2. Amazon SimpleDB, http://aws.amazon.com/simpledb/ 
3. AT\&T Synaptic Storage as a Service, http://www.synaptic.att.com

4. CloudMQ Message Queuing as a Service, http://www.cloudmq.com/

5. Database.com, http://www.database.com/

6. Eucalyptus, The Open Source Cloud Platform, http://open.eucalyptus.com/

7. Haizea, An Open Source VM-based Lease Manager, http://haizea.cs.uchicago.edu/

8. Microsoft .NET Framework, http://www.microsoft.com/net/

9. MONO, Cross platform open source .NET development framework, http://www.mono-project.com/

10. Nimbus Project, http://www.nimbusproject.org/

11. NimbusDB, http://www.nimbusdb.com/

12. Nirvanix Storage Delivery Network, http://www.nirvanix.com/

13. OCCI - Open Cloud Computing Interface, http://occi-wg.org/

14. Open Virtualization Format (OVF) Specification. DSP0243 1.0.0. Distributed Management Task Force (February 2009), http://www.dmtf.org/standards/ovf

15. OpenNebula, The Open Source Toolkit for Cloud Computing, http://www.opennebula.org

16. RabbitMQ Messaging that just works, http://www.rabbitmq.com/

17. Standard ECMA-335 Common Language Infrastructure (CLI), http://www.ecma-international.org/publications/standards/Ecma-335.htm

18. Accenture, Microsoft, WSP: Cloud Computing and Sustainability: The Environmental Benefits of Moving to the Cloud (2011), http://www.zdnet.co.uk/whitepapers/view/server-platforms-os/cloud-computing-and-sustainabilitytheenvironmental-benefits-of-movingto-the-cloud-261937611/2/

19. Bernstein, D., Vij, D.: Using semantic web ontology for intercloud directories and exchanges. In: International Conference on Internet Computing, pp. 18-24 (2010)

20. Dean, J., Ghemawat, S.: MapReduce: Simplified Data Processing on Large Clusters. In: OSDI 2004, pp. 137-150 (2004)

21. Di Martino, B., Petcu, D., Cossu, R., Goncalves, P., Máhr, T., Loichate, M.: Building a Mosaic of Clouds. In: Guarracino, M.R., Vivien, F., Träff, J.L., Cannatoro, M., Danelutto, M., Hast, A., Perla, F., Knüpfer, A., Di Martino, B., Alexander, M. (eds.) Euro-Par-Workshop 2010. LNCS, vol. 6586, pp. 571-578. Springer, Heidelberg (2011), http://dx.doi.org/10.1007/978-3-642-21878-1_70

22. Galán, F., Sampaio, A., Rodero-Merino, L., Loy, I., Gil, V., Vaquero, L.M.: Service specification in cloud environments based on extensions to open standards. In: Proceedings of the Fourth International ICST Conference on Communication System Software and Middleware, COMSWARE 2009, pp. 19:1-19:12. ACM, New York (2009), http://doi.acm.org/10.1145/1621890.1621915

23. Harmer, T., Wright, P., Cunningham, C., Perrott, R.: Provider-Independent Use of the Cloud. In: Sips, H., Epema, D., Lin, H.-X. (eds.) Euro-Par 2009. LNCS, vol. 5704, pp. 454-465. Springer, Heidelberg (2009),

http://dx.doi.org/10.1007/978-3-642-03869-3_44

24. IDC: IDC's Public IT Cloud Services Forecast: New Numbers, Same Disruptive Story, http://blogs . idc . com/ie/?p=922

25. IDC: The Single Biggest Reason Public Clouds Will Dominate the Next Era of IT, http://blogs . idc. com/ie/?p=345

26. Petcu, D.: Identifying cloud computing usage patterns. In: 2010 IEEE International Conference on Cluster Computing Workshops and Posters (CLUSTER WORKSHOPS), pp. 1-8 (September 2010) 
27. Petcu, D., Craciun, C., Rak, M.: Towards a cross-platform cloud api. components for cloud federation. In: 1st International Conference on Cloud Computing \& Services Science, pp. 166-169 (2011)

28. Rochwerger, B., Breitgand, D., Levy, E., Galis, A., Nagin, K., Llorente, I.M., Montero, R., Wolfsthal, Y., Elmroth, E., Caceres, J., Ben-Yehuda, M., Emmerich, W., Galan, F.: The Reservoir model and architecture for open federated cloud computing. IBM Journal of Research and Development 53(4), 4:1 -4:11 (2009)

29. Sheu, P.Y., Wang, S., Wang, Q., Hao, K., Paul, R.: Semantic Computing, Cloud Computing, and Semantic Search Engine. In: IEEE International Conference on Semantic Computing, ICSC 2009, pp. 654-657 (2009)

30. Sun, Y.L., Perrott, R., Harmer, T., Cunningham, C., Wright, P.: An SLA Focused Financial Services Infrastructure. In: International Conference on Cloud Computing \& Virtualization 2010, Singapore (2010)

31. Vecchiola, C., Chu, X., Buyya, R.: Aneka: A Software Platform for .NET-based Cloud Computing, pp. 267-295. IOS Press Inc. (2009) 